\title{
Analysis of 16S Ribosomal DNA Sequences of Francisella Strains and Utilization for Determination of the Phylogeny of the Genus and for Identification of Strains by PCR
}

\author{
MATS FORSMAN, ${ }^{1}$ GUNNAR SANDSTROM, ${ }^{1,2}$ AND ANDERS SJOSTEDT ${ }^{1,2 *}$ \\ Department of Microbiology, National Defence Research Establishment, S-901 82 Umeå, $^{1 *}$ and \\ Department of Infectious Diseases, University of Umeå, S-901 87 Umeå, ${ }^{2}$ Sweden
}

\begin{abstract}
The 16S ribosomal DNAs (rDNAs) of two strains of Francisella tularensis and one strain of Francisella philomiragia were sequenced. On the basis of phylogenetic analysis data, the genus Francisella was placed in the $\gamma$ subclass of the Proteobacteria. The most closely related organism was the intracellular bacterium Wolbachia persica. The sequenced $16 \mathrm{~S}$ rDNA molecules of the Francisella species exhibited very high levels of similarity (98.5 to 99.9\%). Two variable regions, comprising 390 to 450 nucleotides of the $16 S$ rDNA molecules of 17 additional Francisella strains, including members of the species $F$. tularensis and $F$. philomiragia, were also sequenced. At most, six nucleotide differences were observed among the sequences of the $F$. tularensis strains. The sequence of Francisella novicida was virtually identical to the sequences of the $F$. tularensis strains, thereby supporting the hypothesis that these organisms are members of the same species. On the basis of the observed differences, primer pairs were designed to distinguish strains by using the PCR at the genus, species, and subspecies levels. This permitted sensitive identification of strains belonging to the genus Francisella and discrimination of the species $F$. tularensis and $F$. philomiragia.
\end{abstract}

Members of the genus Francisella are identified by biochemical characteristics and an unusual fatty acid composition (6). Two species, Francisella tularensis and Francisella philomiragia, have been recognized, and studies of $F$. tularensis have been responsible for virtually all of our knowledge concerning the genus. This organism is a facultatively intracellular bacterium and the causative agent of the zoonotic disease tularemia (25). There are two biovars of $F$. tularensis (20), and $F$. tularensis biovar tularensis, also designated type $A$, is the predominant biovar found in North America $(20,25)$; this organism is highly virulent in humans $(20,25) . F$. tularensis biovar palaearctica, also designated type B, occurs in Europe, Asia, and North America $(20,25)$; this biovar is less virulent in humans $(5,20,25)$. The two biovars differ in only a limited number of biochemical characteristics, and serological studies have indicated that they are antigenically similar $(15,18,20)$.

$F$. philomiragia is occasionally associated with disease in humans with compromised conditions (12). Its inclusion in the genus Francisella is based on similarities to $F$. tularensis in antigen and fatty acid compositions (12). Until recently, a third species, Francisella novicida, was recognized (6). However, because of phenotypic similarity of $F$. tularensis, the validity of this species has been questioned, and the designation $F$. tularensis biovar novicida has been suggested (12).

Currently used phenotypic criteria do not provide enough information to assess the relationship of $F$. tularensis to other bacterial genera, and the results of DNA hybridization experiments have not documented a close relationship between the genus Francisella and any of several other bacterial genera examined (6).

The lack of phylogenetic placement of the genus Francisella was the reason for undertaking this study. Our approach was to determine the $16 \mathrm{~S}$ ribosomal DNA (rDNA) sequences of members of each of the two Francisella spe-

* Corresponding author. Phone: 4690-106665. Fax: 4690-106800. cies. Phylogenetic analysis based on 16S rRNA and 16S rDNA sequences has been widely used $(2-4,10,11,17-19$, 26).

Moreover, we wanted to determine whether a comprehensive analysis of the variable regions of the $16 \mathrm{~S}$ rDNA molecule of $F$. tularensis would provide information that could clarify the relationship of strains within the species $F$. tularensis. We hoped not only to be able to define more precisely the validity of the species $F$. novicida ( $F$. tularensis biovar novicida), but also to clarify the relationships of various $F$. tularensis strains.

\section{MATERIALS AND METHODS}

Bacteria, media, and growth conditions. The bacterial strains used in this study are listed in Table 1 . These strains are part of our Francisella strain collection (FSC), which contains approximately 100 Francisella strains; strains have been generously donated to us by researchers from several countries. The available information about each strain was documented, and each strain was assigned a strain collection number (Table 1). We characterized all of the strains biochemically and by agglutination. With one exception, the strains used in this study were found to belong to the genus Francisella. The exception was strain FSC 059, which was identified as Staphylococcus warneri after it had been included in this study.

All Francisella strains were grown for 3 days on modified Thayer-Martin medium (22). Cell extracts of virulent Francisella strains (concentration, $10^{9}$ bacteria per ml of saline) were prepared by heat treatment at $65^{\circ} \mathrm{C}$ for $2 \mathrm{~h}$. All other bacterial strains were grown at $37^{\circ} \mathrm{C}$ in Luria-Bertani medium (1).

16S rDNA sequencing. Direct sequencing of amplified DNA was performed by using the method developed by Hultman et al. (13). The 16S rRNA gene to be sequenced was amplified by using prokaryotic 16S rDNA universal primers F1 and R13 (4). This set of primers was used in two separate pairs; in each pair, either F1 or R13 was biotiny- 
TABLE 1. Strains included in this study

\begin{tabular}{|c|c|c|c|c|c|}
\hline $\begin{array}{l}\text { Laboratory } \\
\text { no. }^{a}\end{array}$ & Species & Strain designation(s) ${ }^{b}$ & $\begin{array}{c}\text { Origin } \\
\text { (trivial designation) }^{c}\end{array}$ & $\begin{array}{l}\text { Virulence } \\
\text { for } \\
\text { rabbits }^{d}\end{array}$ & $\begin{array}{l}\text { Nucleotide } \\
\text { sequence } \\
\text { accession no.e }\end{array}$ \\
\hline 1 & Francisella tularensis & FSC 043 & Human, Ohio (SCHU) & High & Z21932 \\
\hline 2 & Francisella tularensis & FSC 033 & Squirrel, Georgia (SnMF) & High & $\mathrm{Z} 22888, \mathrm{Z} 22889$ \\
\hline 3 & Francisella tularensis & FSC 042 & Hare, Canada (Utter) & High & Z22908, Z22909 \\
\hline 4 & Francisella tularensis & FSC 036 & Beaver, Oregon $(0-415)$ & $\mathrm{NP}^{f}$ & \\
\hline 5 & Francisella tularensis & FSC 041 & Tick, Canada (Vavenby) & High & Z22898, Z22899 \\
\hline 6 & Francisella tularensis & FSC 058 & Beaver, Montana (117) & NI & \\
\hline 7 & Francisella tularensis & FSC 045 & Squirrel monkey, United States & NI & \\
\hline 8 & Francisella tularensis & FSC 046 & Fox, Ohio & NI & Z22896, Z22897 \\
\hline 9 & Francisella tularensis & FSC 054 & Tick, Nevada & High & Z22904, Z22905 \\
\hline 10 & Francisella tularensis & FSC 056 & Eigelsbach strain, United States & Low & \\
\hline 11 & Francisella tularensis & FSC 044 & $\begin{array}{l}\text { City water supply, United States } \\
\text { (Helena) }\end{array}$ & Low & Z22902, Z22903 \\
\hline 12 & Francisella tularensis & ATCC 6223, FSC 138 & Human, Utah & Low & Z22912, Z22913 \\
\hline 13 & Francisella tularensis & ATCC 29684, FSC 155 & Live vaccine strain (LVS) & Low & Z21931 \\
\hline 14 & Francisella tularensis & FSC 108 & Human, Sweden (SBL R45) & Low & Z22910, Z22911 \\
\hline 15 & Francisella tularensis & FSC 106 & Human, Sweden (SBL R15) & Low & \\
\hline 16 & Francisella tularensis & CCUG 17299, FSC 146 & Human, Sweden & Low & Z22894, Z22895 \\
\hline 17 & Francisella tularensis & FSC 091 & Human, Norway (9/15) & Low & \\
\hline 18 & Francisella tularensis & FSC 025 & Chatearoux, France & NI & \\
\hline 19 & Francisella tularensis & FSC 026 & Charney, France & NI & Z22921, Z22922 \\
\hline 20 & Francisella tularensis & FSC 151 & Water, Russia (P-13863) & Low & Z22918, Z22919 \\
\hline 21 & Francisella tularensis & FSC 152 & Common vole, Russia (P-13864) & Low & \\
\hline 22 & Francisella novicida & ATCC 15482, FSC 040 & Water, Utah & Low & Z22916, Z22917 \\
\hline 23 & Francisella philomiragia & ATCC 25017, FSC 153 & Human, Utah & & Z21933 \\
\hline 24 & Francisella philomiragia & CCUG 12603, FSC 145 & Human, Sweden & & \\
\hline 25 & Francisella philomiragia & CDC E6588, FSC 154 & Human, Switzerland & & Z22890, Z22891 \\
\hline 26 & Francisella philomiragia & ATCC 25015, FSC 144 & Muskrat, Utah & & $\mathrm{Z} 22900, \mathrm{Z} 22901$ \\
\hline 27 & Staphylococcus warneri & CCUG 30782, FSC 059 & & & \\
\hline 28 & Francisella tularensis & FSC 090 & Human, Japan (Jap4) & Low & Z22914, Z22915 \\
\hline 29 & Francisella tularensis & FSC 017 & Human, Japan (S-2) & Low & \\
\hline 30 & Francisella tularensis & FSC 022 & Human, Japan (Ebina) & Low & Z22906, Z22907 \\
\hline 31 & Francisella tularensis & FSC 149 & Gerbil, Middle Asia, CIS $(120)^{g}$ & Low & \\
\hline 32 & Francisella tularensis & FSC 147 & Hare, Middle Asia, CIS (543) & Low & Z22892, Z22893 \\
\hline 33 & Francisella tularensis & ATCC 29684, FSC 155 & (LVS) & Low & \\
\hline 34 & Staphylococcus aureus & ATCC 6538 & & & \\
\hline 35 & Salmonella typhimurium & ATCC 19585 & & & \\
\hline 36 & Yersinia enterocolitica & ATCC 9610 & & & \\
\hline 37 & Bacillus subtilis & ATCC 6633 & & & \\
\hline 38 & Escherichia coli & ATCC e 23716 & & & \\
\hline 39 & Coxiella burnetii & ATCC VR 146 & & & \\
\hline 40 & Legionella pneumophila & ATCC 33152 & & & \\
\hline 41 & Listeria monocytogenes & ATCC 19111 & & & \\
\hline 42 & Wolbachia persica & ATCC VR 331 & & & \\
\hline
\end{tabular}

a Numbers used to label lanes in Fig. 3 through 5.

$b$ Culture collection designations.

$c$ Trivial designations are designations other than culture collection designations.

${ }^{d}$ As defined in reference 22 .

$e$ EMBL data base and Genbank accession numbers.

$f$ NI, no information.

$g$ CIS, Commonwealth of Independent States.

lated. The starting materials used for DNA amplification were $1-\mu l$ portions of heat-treated Francisella cell extracts. PCRs were performed in a total volume of $50 \mu$ l, essentially as described previously (13). Primers F1 and R13 were used at a concentration of $0.5 \mu \mathrm{M}$, and $3 \mathrm{mM} \mathrm{MgCl}$ was included. Samples were subjected to 30 cycles of amplification in a DNA thermal cycler (model 480; Perkin-Elmer Cetus, Norwalk, Conn.). Each amplification cycle consisted of denaturation for $30 \mathrm{~s}$ at $94^{\circ} \mathrm{C}$, primer annealing to the template at $60^{\circ} \mathrm{C}$ for $1 \mathrm{~min}$, and primer extension at $72^{\circ} \mathrm{C}$ for $1 \mathrm{~min}$. After amplification, the PCR products were purified with a Centricon-3 cartridge (Amicon). Then, the double-stranded PCR products were immobilized on Dynabeads (type M-280 Streptavidin; Dynal A. S., Oslo, Norway) and made single stranded by adding $\mathrm{NaOH}$ (13). Sequence reactions were performed by the dideoxy termination method (23), using Sequenase (USB Corp., Cleveland, Ohio) according to the instructions of the manufacturer. The primers used for sequencing were either nonbiotinylated F1 or nonbiotinylated R13 primer and nine other primers synthesized on the basis of the previously sequenced regions of the Francisella 16S rRNA molecule (9), scattered around the whole Francisella 16S rRNA gene. Both strands were sequenced at least once in every position. Additional primers were also designed in order to sequence two variable regions of the $16 \mathrm{~S}$ rRNA genes of 17 additional Francisella strains. These primers, designated FT9 (5'-CGGCCCAAACTCCTACG GGA-3') and FT10 (5'-AAGTCCCGCAACGAGCGCAA- 
TABLE 2. Positions of the primers used in the PCR

\begin{tabular}{llll}
\hline Primer & \multicolumn{1}{c}{ Position $^{a}$} & \multicolumn{1}{c}{ Sequence } & Reference \\
\hline F1 & $11-29$ & 5'-GAGTTTGATCCTGGCTCAG-3' & 4 \\
R13 & $1544-1525$ & 5'-AGAAAGGAGGTGATCCAGCC-3' & 4 \\
F5 & $1290-1272$ & 5'-CCTTTTTGAGTTTCGCTCC-3' & This study \\
F11 & $149-168$ & 5'-TACCAGTTGGAAACGACTGT-3' & This study \\
FP5 & $1290-1272$ & 5'-CCTTTCTGAGTTTCGCTCC-3' & This study \\
FP8 & $457-478$ & 5'-ATGAGTTAATAGCTTGTAGGAA-3' & This study \\
FTS8 & $457-478$ & 5'-CAAGGTTAATAGCCTTGGGGA-3' & This study \\
FTS12 & $1171-1152$ & 5'-CCTTGTCAGCGGCAGTCTCA-3' & This study \\
FTL8 & $457-478$ & 5'-CAAGGTTAATAGCCTTGGGGGA-3' & This study \\
FTL12 & $1172-1152$ & 5'-GCCTTGTCAGCGGCAGTCTTA-3' & This study \\
\hline
\end{tabular}

${ }^{a}$ Escherichia coli numbering.

$3^{\prime}$ ), were located at positions corresponding to positions 329 to 348 and 1110 to 1091, respectively, on the Escherichia coli $16 \mathrm{~S}$ rRNA gene.

After completion of the termination reaction, the reaction mixtures were heated at $65^{\circ} \mathrm{C}$ for $5 \mathrm{~min}$. The supernatants containing the newly synthesized DNA strands were removed, and 2.5- $\mu$ l portions of the samples were electrophoresed in a $6 \%$ polyacrylamide- $7 \mathrm{M}$ urea gel.

PCR identification of Francisella strains. One-microliter portions of heat-treated cell extracts of the virulent Francisella strains (corresponding to approximately $10^{6}$ bacterial cells) were used in the PCR. Wolbachia persica ATCC VR331 was obtained lyophilized. The material was resuspended in $1 \mathrm{ml}$ of saline, $0.1 \mathrm{ml}$ of this preparation was treated by the alkaline lysis method as described previously (21) and the resulting material was resuspended to a volume of $10 \mu \mathrm{l} ; 1 \mu \mathrm{l}$ of this preparation was used for each PCR.

The conditions used for the PCR were similar to those described above. A preheating cycle at $94^{\circ} \mathrm{C}$ for $7 \mathrm{~min}$ was included, and the optimum $\mathrm{MgCl}_{2}$ concentration $(1.5 \mathrm{mM})$ was used. Both $T a q$ polymerase and primers were added during the preheating. The primers used are listed in Table 2. The amplification cycles used for denaturation, primer annealing, and primer extension were as follows: $94^{\circ} \mathrm{C}$ for 1 $\mathrm{min}, 65^{\circ} \mathrm{C}$ for $1 \mathrm{~min}$, and $72^{\circ} \mathrm{C}$ for $1 \mathrm{~min}$ for the F5-F11 primer pair; $94^{\circ} \mathrm{C}$ for $1 \mathrm{~min}, 62^{\circ} \mathrm{C}$ for $1 \mathrm{~min}$, and $72^{\circ} \mathrm{C}$ for $1 \mathrm{~min}$ for the FP5-FP8 primer pair; and $94^{\circ} \mathrm{C}$ for $1 \mathrm{~min}$ and $68^{\circ} \mathrm{C}$ for 1 min (only two temperatures) for the FTL8-FTL12 and FTS8FTS12 primer pairs and combinations of these primer pairs. After 35 cycles of amplification, 5- $\mu$ l portions of the PCR products were electrophoresed in a $1.0 \%$ agarose gel, stained with ethidium bromide, and photographed.

Data analysis. The 16S rDNA sequences of the species indicated in Table 3 were aligned and compared to determine similarity. Only positions that were identical in at least $50 \%$ of the species and positions that did not overlap with gaps in the alignment were included in the analysis. These constraints reduced the number of positions to 1,327 . The levels of similarity between the sequences were converted to nucleotide substitution values $(7,14)$. These values were used to produce a phylogenetic tree by the method of Fitch and Margoliash (8). Statistical confidence in the topology was assessed by using SEQBOOT, DNADIST, FITCH, and CONSENSE in succession, as executed by the PHYLIP software package (version 3.5) (7).

The signature positions (Table 4) for the genus Francisella were identified by comparing the 16S rDNA sequences of $F$. tularensis SCHU and LVS, $F$. philomiragia, and $W$. persica with the 16S rRNA sequences of Oceanospirillum linum, Ectothiorhodospira shaposhnikovi, and the bacteria listed in Table 3, 21 16S rRNA sequences compiled by Dams et al. (2), and the sequences of 54 members of the family Pasteurellaceae (3).

\section{RESULTS}

Sequence determination. Virtually complete $16 \mathrm{~S}$ rDNA sequences $(1,518$ to 1,521 nucleotides) for the following Francisella strains were determined: (i) a strain belonging to the species $F$. philomiragia (ATCC 25017), (ii) the live vaccine strain $F$. tularensis LVS (ATCC 29684), a type B strain from Russia, and (iii) the highly virulent strain SCHU, a typical type A strain (15). The sequences have been deposited in the EMBL and GenBank nucleotide sequence data bases.

$F$. tularensis SCHU exhibited $99.9 \%$ similarity to $F$. tularensis LVS and $99.2 \%$ similarity to $F$. philomiragia. Our analysis demonstrated that 14 of the 26 differences between

TABLE 3. Levels of sequence similarity and evolutionary distances based on alignment of 1,327 nucleotides of 16S rDNAs from Francisella species, W. persica, and some reference species belonging to the $\gamma$ subclass of the Proteobacteria ${ }^{a}$

\begin{tabular}{|c|c|c|c|c|c|c|c|c|c|}
\hline \multirow[b]{2}{*}{ Species } & \multicolumn{9}{|c|}{$\%$ Sequence similarity or evolutionary distance ${ }^{b}$} \\
\hline & 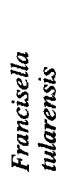 & 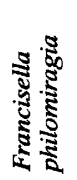 & 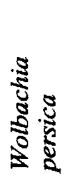 & 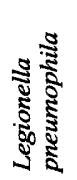 & 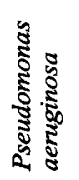 & 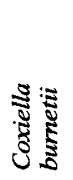 & 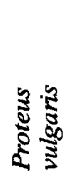 & 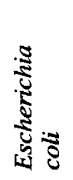 & 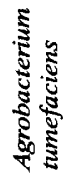 \\
\hline Francisella tularensis & & 99.2 & 97.8 & 88.7 & 87.9 & 87.3 & 85.5 & 85.8 & 82.9 \\
\hline Francisella philomiragia & 0.8 & & 97.4 & 88.5 & 87.7 & 87.4 & 85.5 & 85.8 & 82.9 \\
\hline Wolbachia persica & 2.2 & 2.6 & & 88.5 & 88.1 & 87.0 & 85.1 & 85.3 & 83.3 \\
\hline Legionella pneumophila & 12.6 & 12.9 & 12.9 & & 90.3 & 90.5 & 87.5 & 87.4 & 83.7 \\
\hline Pseudomonas aeruginosa & 13.4 & 13.7 & 13.3 & 10.7 & & 90.3 & 87.6 & 88.8 & 83.6 \\
\hline Coxiella burnetii & 14.2 & 14.1 & 14.8 & 10.4 & 10.6 & & 86.5 & 87.8 & 84.0 \\
\hline Proteus vulgaris & 16.6 & 16.6 & 17.2 & 14.1 & 13.8 & 15.2 & & 95.0 & 82.6 \\
\hline Escherichia coli & 16.3 & 16.3 & 17.0 & 14.3 & 12.4 & 13.7 & 5.2 & & 82.2 \\
\hline Agrobacterium tumefaciens & 20.1 & 20.1 & 19.7 & 19.1 & 19.2 & 18.7 & 20.7 & 21.3 & \\
\hline
\end{tabular}

${ }^{a}$ Evolutionary distances were calculated as described in Materials and Methods. The EMBL accession numbers for sequences used in the alignment are as follows: Agrobacterium tumefaciens, M11223; Coxiella burnetii, M21291; Escherichia coli, J01859; Legionella pneumophila, M59157; Proteus vulgaris, X07652; Pseudomonas aeruginosa, M34133; and Wolbachia persica, M21292.

$b$ The values on the upper right are levels of sequence similarity, and the values on the lower left are evolutionary distances. 
TABLE 4. Sequence signatures for the genus Francisella and $W$. persica

\begin{tabular}{|c|c|c|c|}
\hline \multirow{2}{*}{ Position $^{a}$} & \multicolumn{2}{|c|}{ Nucleotide in: } & \multirow{2}{*}{ Exceptions $^{d}$} \\
\hline & Francisella $^{b}$ & $\overline{\text { Eubacteria }}{ }^{c}$ & \\
\hline 40 & $\mathrm{U}$ & $\mathrm{C}$ & \\
\hline 151 & $\mathrm{C}$ & $A, g$ & $\begin{array}{l}\text { Chloroflexus auranticus, } \\
\text { Oceanospirillum linum }\end{array}$ \\
\hline 294 & $\mathbf{U}$ & $\mathrm{C}$ & \\
\hline 336 & A & $\mathbf{G}$ & Herpetosiphon aurantiacus \\
\hline 401 & A & G & \\
\hline 402 & $\mathbf{U}$ & $\mathrm{C}$ & \\
\hline 849 & $\mathrm{C}$ & $\mathrm{U}, \mathrm{g}$ & Streptomyces coelicolor \\
\hline 889 & $\mathrm{C}$ & $G^{\circ}$ & Herpetosiphon aurantiacus \\
\hline 1280 & A & $\mathrm{U}, \mathrm{c}$ & $\begin{array}{l}\text { Ruminobacter amylophilus, } \\
\text { Proteus vulgaris }\end{array}$ \\
\hline 1290 & G & $\mathrm{C}, \mathrm{u}$ & Chloroflexus aurantiacus \\
\hline 1453 & $\mathbf{A}$ & G & \\
\hline 1455 & $\mathrm{U}$ & $\mathrm{A}, \mathrm{g}$ & \\
\hline
\end{tabular}

${ }^{a}$ Escherichia coli numbering.

${ }^{b}$ Bases found in Francisella species, including $W$. persica.

${ }^{c}$ Bases found in other members of the Eubacteria. Lowercase letters indicate bases found in less than $25 \%$ of the strains of Eubacteria that have been examined.

${ }^{d}$ Bacterial species having the same base as Francisella strains. the $F$. philomiragia and $F$. tularensis sequences were located within one variable region of the gene, nucleotides 375 to 526 ( $E$. coli numbering). Moreover, a cluster of four differences was identified in another region, nucleotides 207 to 215 .

Sequence similarity analysis. The sequences of Francisella strains were compared with previously determined $16 \mathrm{~S}$ rRNA sequences of prokaryotes included in the EMBL data base (version 32). The signatures (determined by the method of Stackebrandt et al. [24]) of the sequences beginning at positions $168,310,506$, and 1405 were almost identical to the consensus signatures of the $\gamma$ subclass of Proteobacteria. Only one deviation was found; the $C$ at position 168 in Francisella strains was replaced with a U. Signature positions for the genus Francisella were determined (Table 4).

To determine where to place the genus Francisella within the $\gamma$ subclass, levels of similarity to other genera were calculated. Interestingly, the sequence of $W$. persica exhibited $97.4 \%$ similarity to the Francisella sequences (Table 3 ), which was the highest level of similarity observed with any of the sequences included in the data base. A phylogenetic tree (Fig. 1) was constructed. Proteus vulgaris and E. coli were included as reference strains of the $\gamma$ subclass, and Agrobacterium tumefaciens was included as an outgroup. The other strains included were those that exhibited the highest levels of similarity to Francisella strains.

Determination of the sequences of the variable regions of Francisella strains. To assess the relationships among vari-

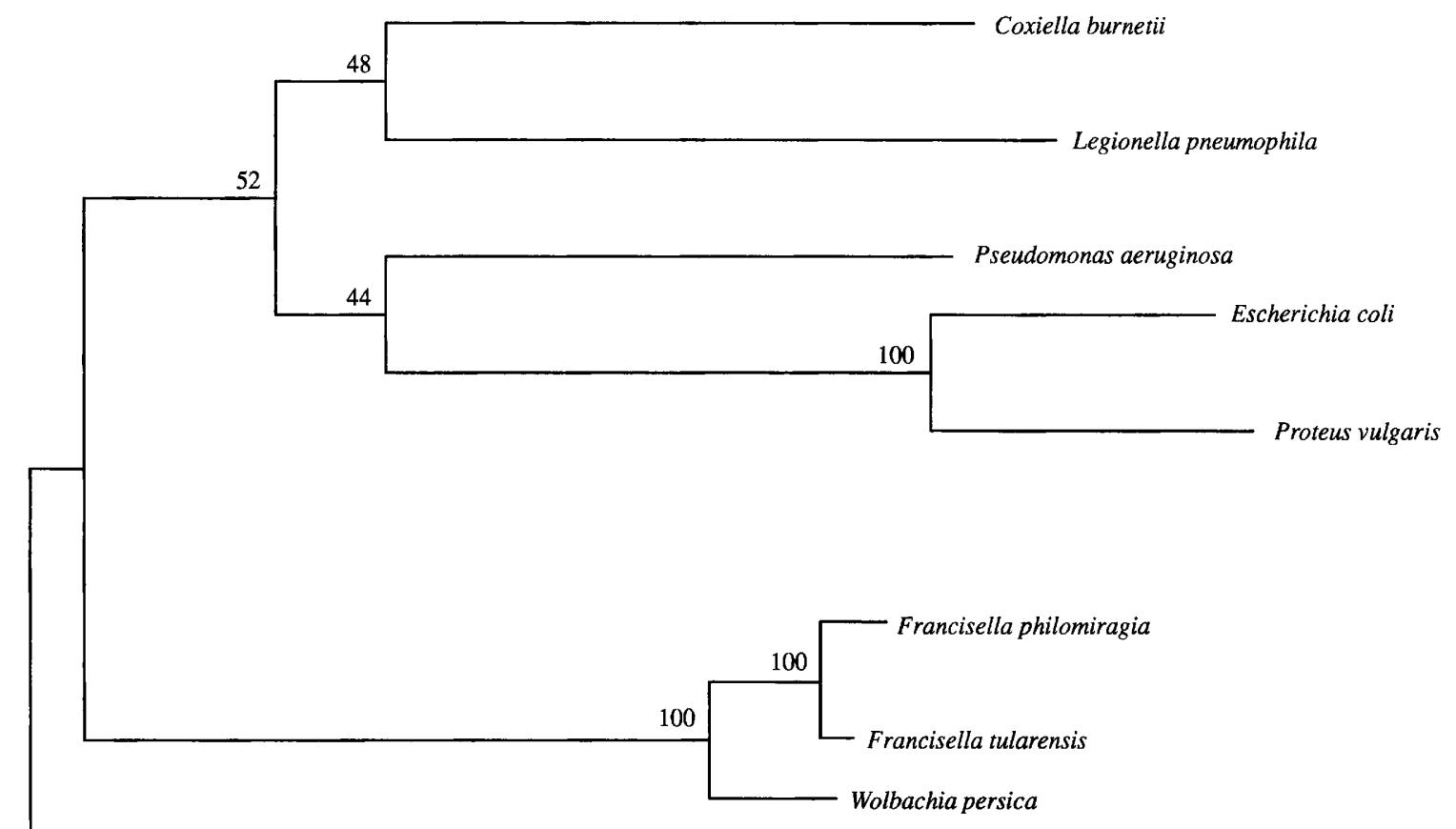

Agrobacterium tumefaciens $1 \%$

FIG. 1. Evolutionary distance tree showing relationships among the organisms used in this study and members of the $\gamma$ subclass of the Proteobacteria that exhibit the highest levels of similarity to members of the genus Francisella. Agrobacterium tumefaciens was included as an outgroup. The unrooted tree was constructed by the algorithm of Fitch and Margoliash after evolutionary distances had been calculated from nucleotides substitution values. The numbers at the nodes of branches are the confidence values (expressed as percentages) obtained from 100 replications. Bar $=$ sequence similarity difference of $1.0 \%$. 
TABLE 5. 16S rRNA signature nucleotides of the different clusters of $F$. tularensis

\begin{tabular}{lcccc}
\hline \multicolumn{1}{c}{ F. tularensis strain(s) } & \multicolumn{4}{c}{ Nucleotide at position: } \\
\cline { 2 - 5 } & 477 & 1153 & $1174 / 385$ & 1292 \\
\hline $\begin{array}{l}\text { SCHU, FSC 033, FSC 041, } \\
\quad \text { FSC 046 }\end{array}$ & $\Delta^{a}$ & G & G/C & A \\
$\begin{array}{l}\text { FSC 042, FSC 054, FSC 138, } \\
\text { FSC 022 }\end{array}$ & G & G & G/C & A \\
LVS, FSC 026, FSC 044, FSC & G & A & G/C & A \\
$\quad$ 108, FSC 146, FSC 151 & G & G & $\Delta / \Delta$ & A \\
FSC 040, FSC 090 & G & G & G/C & G \\
FSC 147
\end{tabular}

${ }^{a} \Delta$, deletion at that position.

${ }^{b}$ Strain FSC 040 has also been identified as $F$. novicida.

ous Francisella strains, 16S rDNAs of 17 additional strains were sequenced; these strains included strains having different phenotypic traits and strains obtained from different geographical areas. The sequences of two regions of the $16 \mathrm{~S}$ rDNAs from positions 375 and 1139 were determined; each of these regions was 170 to 250 nucleotides long. The first region exhibited the highest level of diversity in the alignment of the three complete sequences, whereas the second region was selected because it encompassed nucleotide 1153, which has previously been demonstrated by $16 \mathrm{~S}$ rRNA sequencing and hybridization to vary among Francisella strains $(9,22)$. Of the 26 differences between the complete sequence of $F$. philomiragia (ATCC 25017) and the $F$. tularensis sequences, 16 were located within these two regions. The sequences of the $F$. philomiragia strains differed at six nucleotides. The $F$. tularensis strains exhibited variations at five positions, yielding clusters of strains (Table 5).

The first cluster, distinguished by a deletion at position 477 , contained strains isolated in North America. Three of these strains were highly virulent when they were isolated (Table 1). The second cluster also comprised strains from North America, two of which were highly virulent when they were isolated. Also, avirulent strain B38, which was highly virulent when it was originally isolated (15), was a member of this cluster. The third cluster, which was characterized by an $\mathrm{A}$ at position 1153, contained strains isolated in Europe. The fourth cluster contained $F$. tularensis 090 (a strain isolated in Japan) and $F$. novicida. This may indicate that within the genus Francisella, the phenotypic markers that distinguish $F$. tularensis and $F$. novicida (12) are not necessarily reflected as differences in the 16S rRNA sequences. Actually, the sequence of $F$. novicida was very closely related to all of the $F$. tularensis sequences; within the variable region at positions 375 to 546 at most four differences were found (Fig. 2). Altogether, 743 bp (positions 375 to 642,819 to 929 , and 1036 to 1402 ) of the 16 S rRNA gene of $F$. novicida was sequenced. No differences were found except those in the region from position 375 to position 546 . The fifth cluster contained a strain isolated in the central Asian portion of the former Commonwealth of Independent States.
Besides the differences indicated in Table 5, only one additional deviation was found in the 16S rDNA sequences; $F$. tularensis FSC 146, which originally was isolated from a Swedish patient with meningitis, had a $U$ at position 490 (Fig. 2).

Detection of the genus Francisella. Oligonucleotides were designed for specifically identifying strains belonging to the genus Francisella. On the basis of the sequences determined, two primers, designated F5 and F11, located at positions 1290 to 1272 and 149 to 168 , were selected (Fig. 3A). All 31 Francisella strains but none of eight strains belonging to other genera yielded the appropriate amplified PCR product. In addition to $F$. tularensis $16 \mathrm{~S}$ rDNA, the $16 \mathrm{~S}$ rDNA of $W$. persica was amplified, a finding that was anticipated as the sequences of the two species are identical in the primer-complementary regions. We found no primers that distinguished all of the strains belonging to the genus Francisella from $W$. persica. We also confirmed that all preparations contained DNA that could be amplified, since the F1-R13 primer pair, which was specific to regions conserved in all $16 \mathrm{~S}$ rDNAs, yielded a fragment of the expected size (Fig. 3B).

Identification of Francisella species. Species-specific regions in the two variable regions were identified, and a number of different primer pairs were assessed for usefulness for discriminating these variable sequences. The FTS8FTS12 and FTL8-FTL12 primer pairs, used in equal amounts, specifically amplified the $16 \mathrm{~S}$ rDNAs of all $F$. tularensis strains, including $F$. novicida (Fig. 4A). The FP5-FP8 primer pair was found to react with the four strains of $F$. philomiragia analyzed, but not with $F$. tularensis or any other bacterial species investigated (Fig. 4B).

Differentiation of strains within the species $F$. tularensis. The usefulness of the PCR for identifying different clusters of $F$. tularensis was also assessed. To do this, primers that amplified a fragment encompassing positions 457 to 1172 of the $16 \mathrm{~S}$ rDNA sequence were used. The FTS8-FTS12 primer pair specifically amplified the 16S rDNA of four Francisella strains that lacked a G at position 477 (Fig. 5), three of which were highly virulent when they were isolated. Conversely, the FTL8-FTL12 primer pair identified all of the strains that were not detected by the FTS8-FTS12 primer pair (data not shown).

\section{DISCUSSION}

DNA-DNA hybridization is the standard method used to define bacterial species and, together with phenotypic characteristics, effectively delineates bacterial taxa. Phylogenetic parameters alone do not provide sufficient information for taxonomic evaluation, since evolutionary rates differ among various groups of bacteria. Nonetheless, rRNA analysis is thought to be the only valid method for assessing the present taxonomy. rRNA sequences are considered to be reliable phylogenetic indicators as their variability reflects evolutionary mutations that occur at a relatively regular pace. The variable regions of the molecule include a sufficient number of bases to provide adequate information for analysis of close as well as distant relationships among

FIG. 2. Sequences of the variable regions of the $16 \mathrm{~S}$ rDNA molecules of Francisella strains. The sequences of two regions, positions 375 to 546 (A) and positions 1139 to 1363 (B), of the 16S rDNA molecules were determined. The sequence of $F$. tularensis SCHU FSC 043 is shown, and the nucleotides of the other sequences that differ from the nucleotides in the strain SCHU sequence are indicated. See Table 1 for strain designations. $\Delta$, deletion at that position. 
A

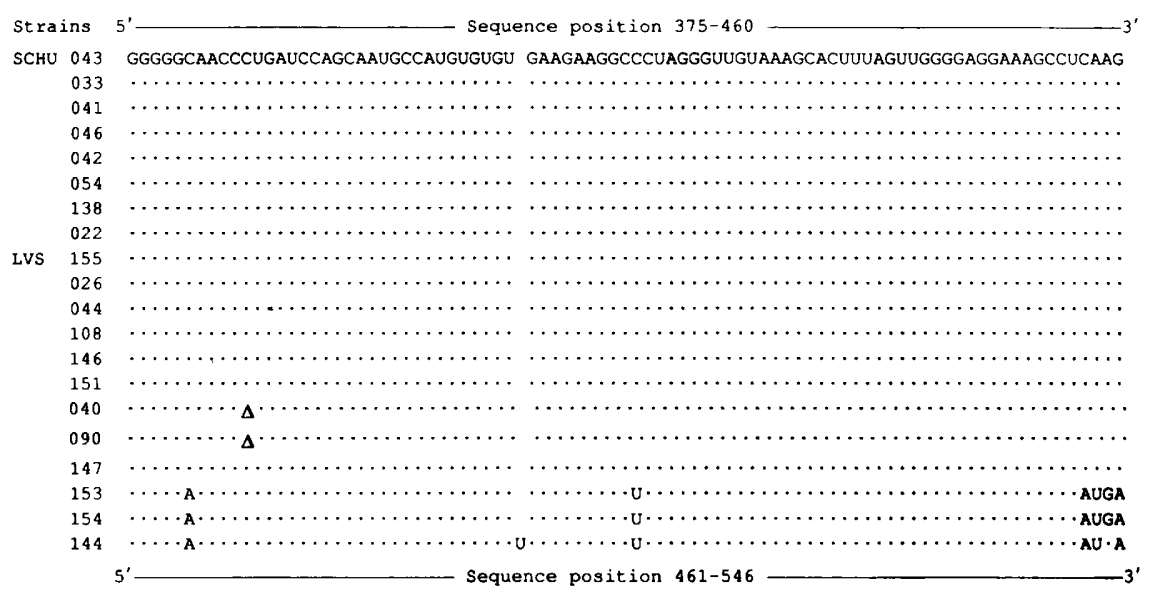

SCHU 043 GUUAAUAGCCUUGGG AGG ACGUUACCCAAAGAAUAAGCACCGGCUAACUCCGUGCCAGCAGCCGCGGUAAUACGGGGGGUGCAA

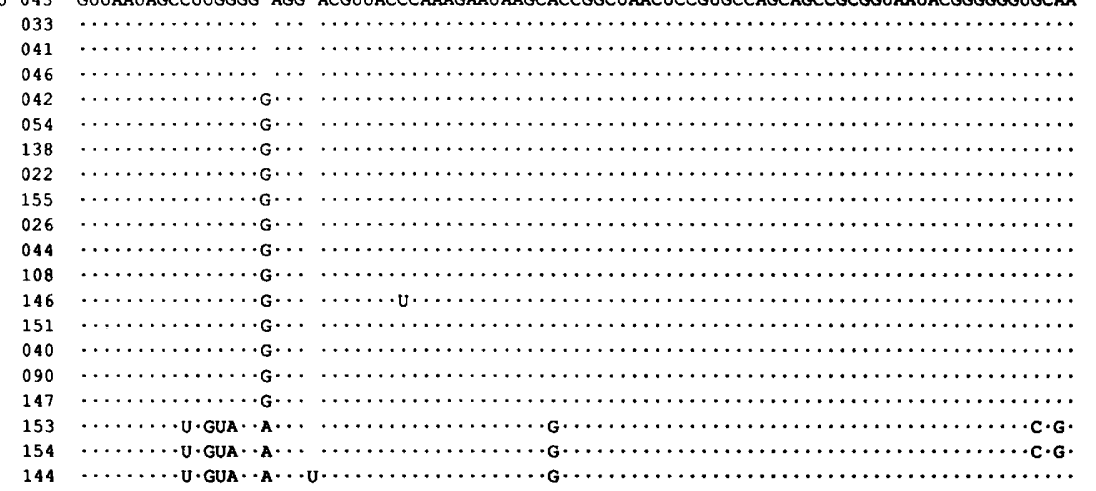

$\mathbf{B}$

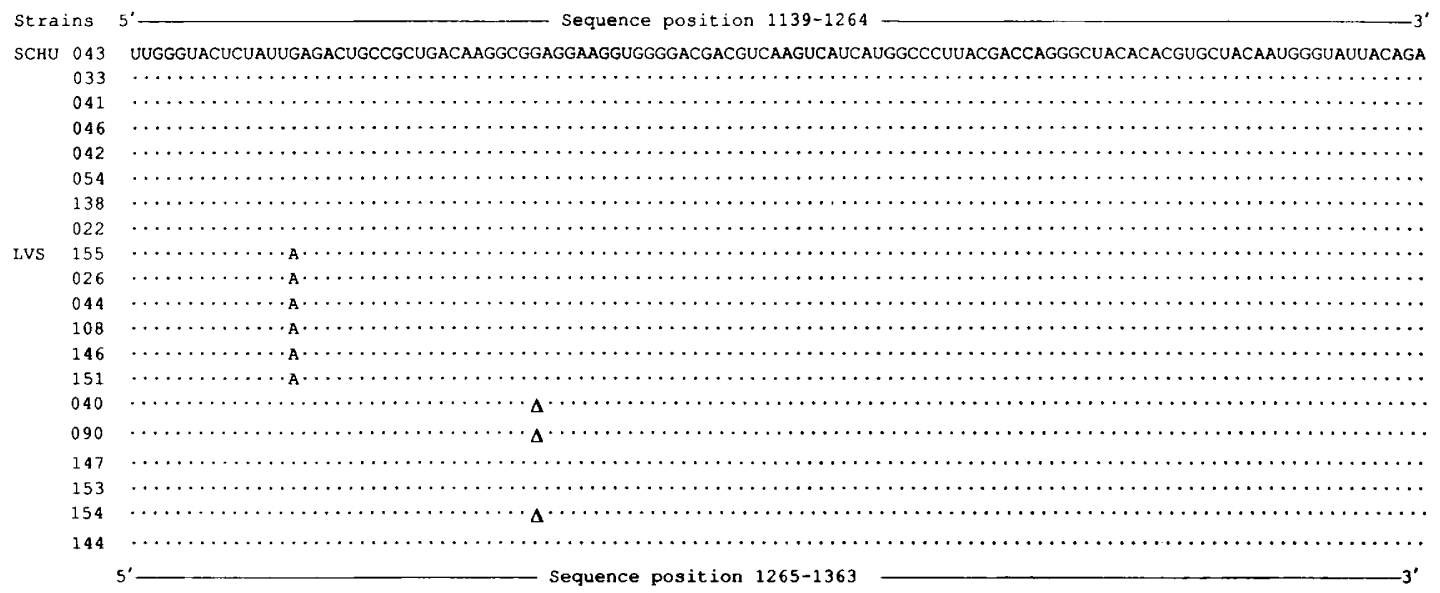

SCHU 043 GGGCUGCGAAGGUGCGAGCUGGAGCGAAACUCAAAAAGGUACUCUUAGUCCGGAUUGCAGUCUGCAACUCGACUGCAUGAAGUCGGAAUCGCUAGUAAUCGCAGGUCAGAAU

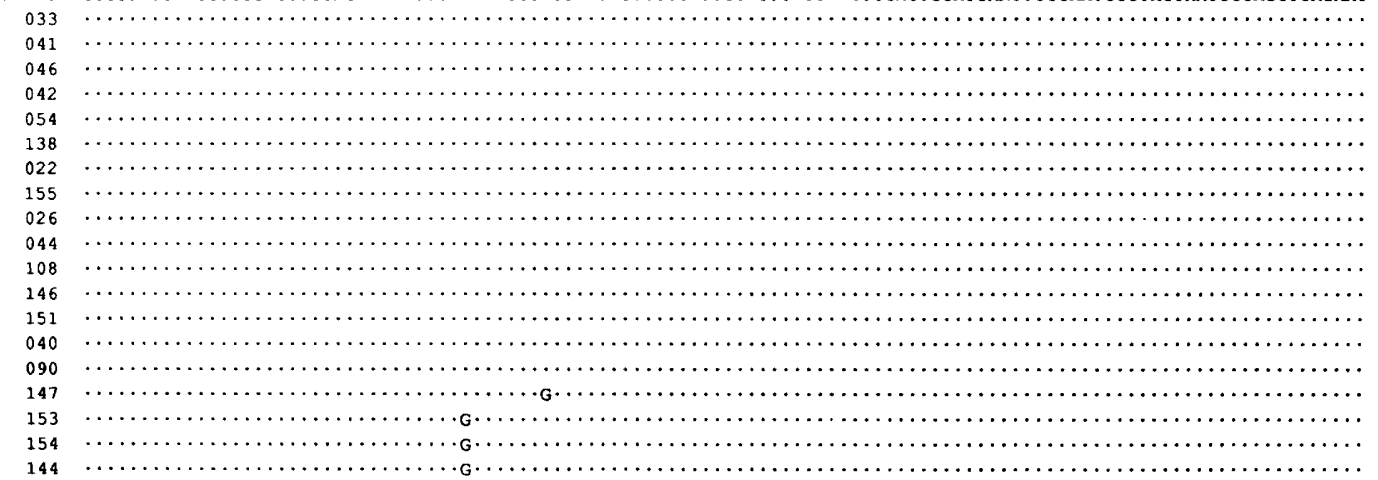



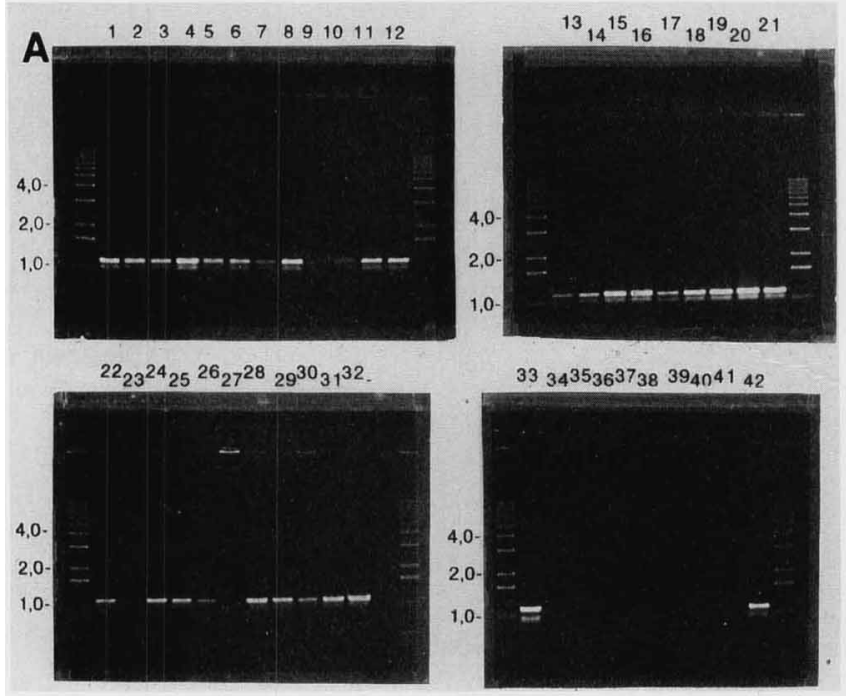

$334^{35} 366^{37} 38 \quad 39404142$
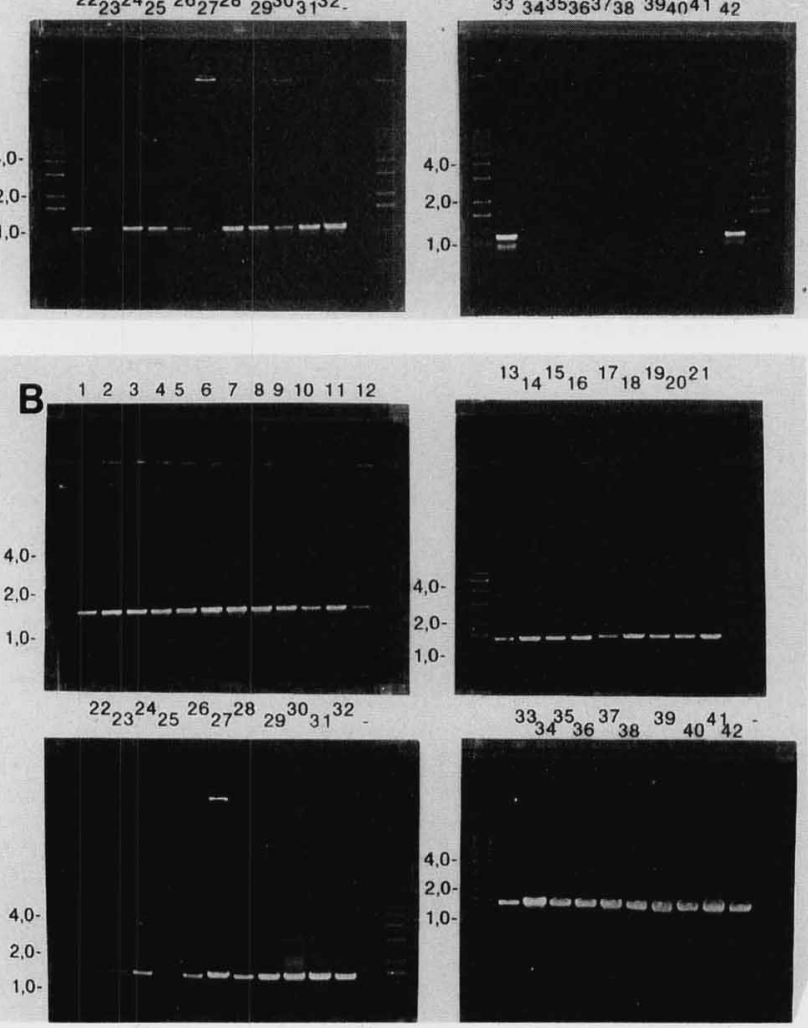

FIG. 3. Results of PCR-assisted amplification of whole cells by the Francisella-specific F5-F11 primer pair (A) and the F1-R13 primer pair complementary to conserved regions in all eubacterial 16S rRNA sequences (B). See Table 1 for an explanation of the bacterial strain numbers above the lanes. The chromosomal DNA from approximately $10^{6}$ bacteria was subjected to 35 cycles of amplification. The resulting PCR product was then electrophoresed in an agarose gel, stained with ethidium bromide, and photographed.

species. Generally, good agreement has been found between previously existing taxonomic groups and phylogenetic data, inasmuch as $95 \%$ of all genera examined exhibit perfect concordance (19). Generally, the majority of recognized species that have been examined differ in their 16S rRNA sequences from related species in at least $1.5 \%$ or more of the positions (10).

An interesting observation in this analysis was the unusually high levels of homology between the 16S rRNA sequences of $W$. persica and Francisella strains. $W$. persica, an obligately intracellular microorganism, has previously been referred to the tribe Rickettsieae, but it has been noted that the phenotype of this organism does not closely resemble the phenotypes of other members of the genus Wolbachia (27). Recently, when its 16S rRNA sequence was determined by Weisburg et al., $W$. persica was found to be
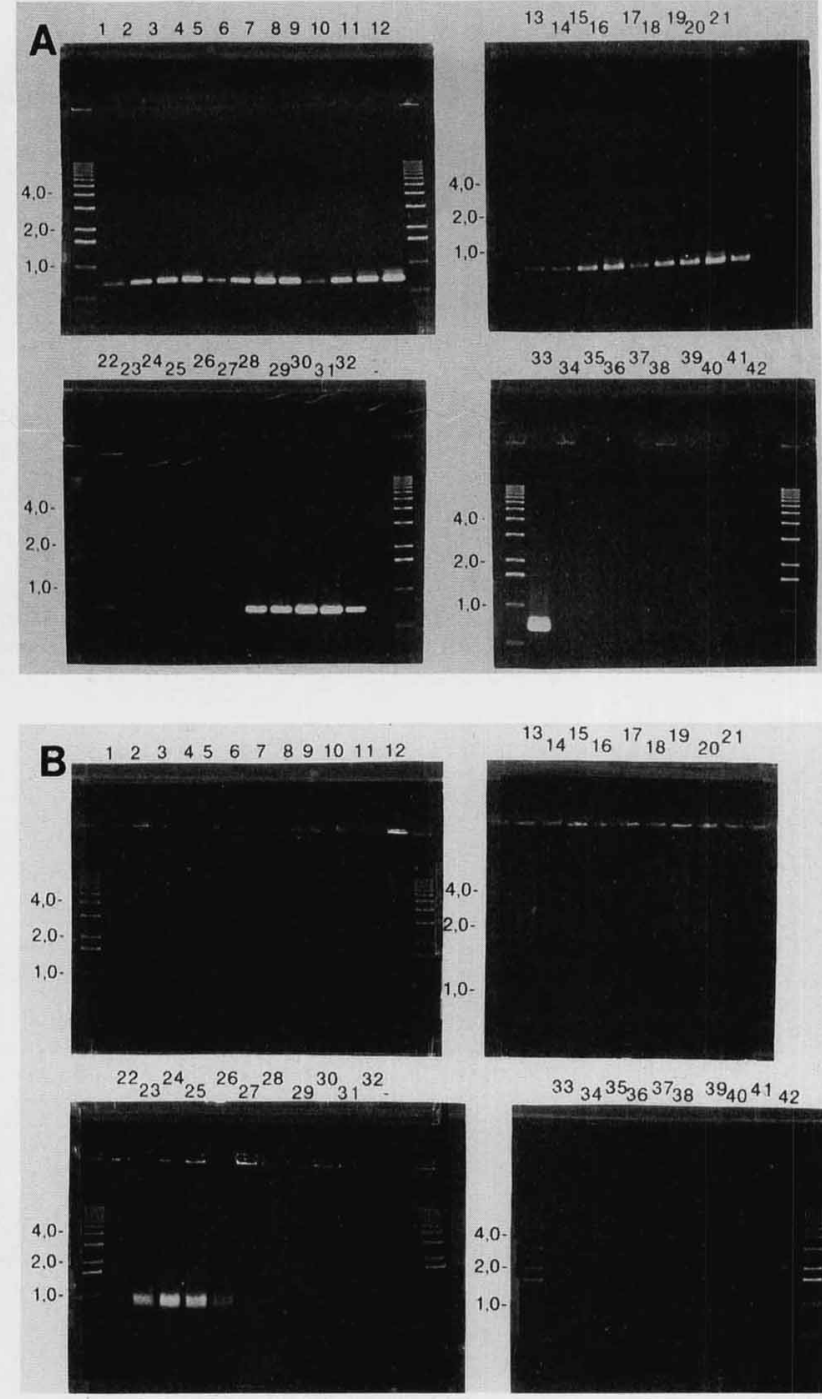

${ }^{33} 34^{35_{36}}{ }^{37} 7_{38}{ }^{39} 9_{40} 4142$

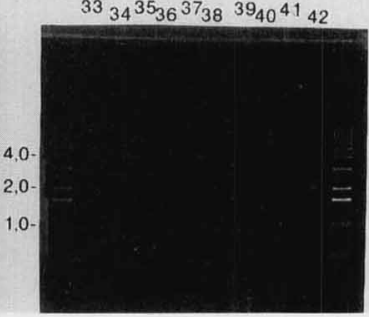

FIG. 4. Results of PCR-assisted amplification of whole cells by the $F$. tularensis-specific FTS8-FTS12 and FTL8-FTL12 primer pairs (A) and the $F$. philomiragia-specific FP5-FP8 primer pair. See Table 1 for an explanation of the bacterial strain numbers above the lanes. The chromosomal DNA from approximately $10^{6}$ bacteria was subjected to 35 cycles of amplification. The resulting PCR product was then electrophoresed in an agarose gel, stained with ethidium bromide, and photographed.

most similar to members of the Coxiella-Legionella cluster (30) and not related to other Wolbachia species or any other species of Rickettsieae. The most notable difference in topology between this study and that of Weisburg et al. is the branching of $W$. persica (Fig. 1). This may have resulted from the different methods used for phylogenetic analysis, the distant relationships of the bacteria included, the low confidence values for the positioning of the Coxiella-Legionella cluster, or the outgroups included.

Our results show that $W$. persica is much more similar to Francisella species than to any other species (Table 3 ). This finding is also supported by the high confidence values $(100 \%)$ obtained (Fig. 2). It should be remarked that the degree of similarity (97.4 to $97.8 \%$ ) does not mean that $W$. persica should be placed in the genus Francisella, but rather 


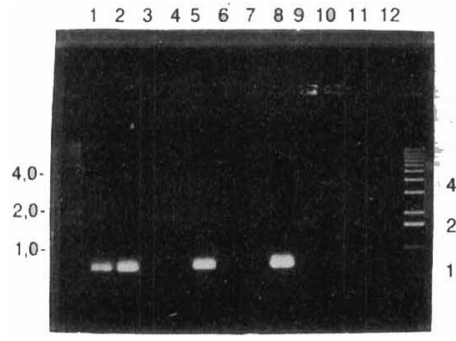

${ }^{22} 23^{24} 25^{26} 27^{28} 29^{30} 31^{32}$
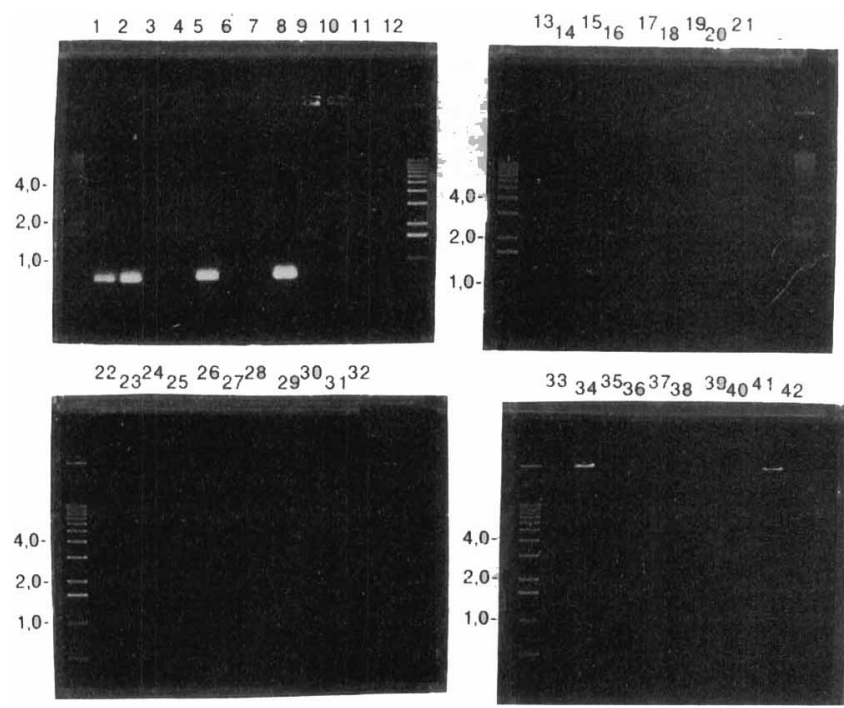

$33_{34}{ }^{35_{36}}{ }^{37} 7_{38} \quad 39_{40} 4^{4} 1_{42}$

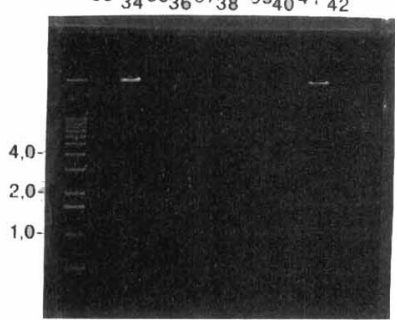

FIG. 5. Results of PCR-assisted amplification of whole cells by the FTS8-FTS12 primer pair. See Table 1 for an explanation of the bacterial strain numbers above the lanes. The chromosomal DNA from approximately $10^{6}$ bacteria was subjected to 35 cycles of amplification. The resulting PCR product was then electrophoresed in an agarose gel, stained with ethidium bromide, and photographed.

does indicate that the species should be further characterized phenotypically to determine its relationship to Francisella species. The high level of similarity of the sequences complicated the search for a Francisella-specific primer pair. Indeed, all primer pairs that could be used to distinguish members of the genus Francisella from other bacteria also reacted with $W$. persica.

The data obtained for the 16S rDNA sequences of the strains belonging to the genus Francisella seem to corroborate the present differentiation of the genus into the species $F$. tularensis and $F$. philomiragia. Another observation is that, despite the clear distinction between the two species, the level of sequence homology $(99.2 \%)$ between $F$. philomiragia and $F$. tularensis is actually higher than the levels of sequence homology found between many species belonging to the same genus (17). Moreover, our results also strengthen the previous suggestion, based on phenotypic data, that $F$. novicida should be classified as a subspecies of F. tularensis (12), as the degree of similarity is $99.6 \%$.

Little is known about other intraspecies variations of $16 \mathrm{~S}$ rRNA sequences. Like our results for Francisella strains a study of the 16S rRNA sequences of Legionella pneumophila strains revealed very little diversity (11). In contrast, up to $1.0 \%$ divergence was demonstrated in a study of Borrelia burgdorferi strains (16). It would be interesting to consider whether the few differences that exist in the $16 \mathrm{~S}$ rRNA sequences of Francisella strains are reflected by differences in geographical distribution or in phenotypic traits, such as virulence.

In accordance with previous findings, $F$. tularensis strains could be divided into two groups depending on the nucleotide present at position $1153(9,22)$. All European strains had an $\mathrm{A}$ in this position, whereas strains isolated outside Europe, with one exception, $F$. tularensis FSC 044, had a G (Fig. 2). However, in the non-European group the strains were phenotypically heterogeneous, as they had different fermentation patterns and exhibited different degrees of virulence (22). At least in this respect, the nucleotide at position 1153 did not correlate with a phenotypic trait.

Identification of Francisella strains by the PCR has advantages compared with methods used previously to identify these bacteria (e.g., biochemical property and fatty acid composition analyses, which are laborious and time consuming). An additional advantage of using the PCR is that the limited diversity found in the $16 \mathrm{~S}$ rRNA sequences of Francisella strains should permit rapid screening of the genotypes of new isolates. If a strict correlation between genotype and phenotype is found in future studies, the PCR would be a suitable method for classifying new isolates.

In conclusion, our study took advantage of the technique of sequencing $16 \mathrm{~S}$ rRNA (rDNA) as a powerful method for establishing bacterial phylogeny and validating previously described taxa. Our phylogenetic analysis confirmed the validity of the present taxonomy of the genus Francisella. In addition, the signature positions of the 16S rDNA sequences at the genus, species, and subspecies levels can be used for rapid and specific identification of Francisella strains by the PCR.

\section{ACKNOWLEDGMENTS}

We thank S. Stewart for kindly supplying most of the Francisella strains included in this study. Strains were also generously donated by E. Falsen, B. Berdal, B. Mishankin, and N. Pavlovich. Advice was given by E. Falsen, D. Brenner, and E. Stackebrandt. We also thank A. Tärnvik for critically reading the manuscript, $H$. Renström for help with the computer analysis, G. Boström for help with the illustrations, and Anna Wiklund and Ulla Eriksson for excellent technical assistance.

This work was supported in part by grants from the Swedish Medical Research Council (project 9485), the Swedish Society for Medical Research, Karolinska Institute, Stockholm, Sweden, and the Swedish Society of Medicine.

\section{REFERENCES}

1. Bertani, G. 1951. Studies on lysogenesis. I. The mode of phage liberation by lysogenic Escherichia coli. J. Bacteriol. 62:293300.

2. Dams, E., L. Hendriks, Y. Van de Peer, J.-M. Neefs, G. Smits, I. Vandenbempt, and R. de Wachter. 1988. Compilation of small ribosomal subunit RNA sequences. Nucleic Acids Res. Suppl. 16:v87-v173.

3. Dewhirst, F. E., B. J. Paster, I. Olsen, and G. J. Fraser. 1992. Phylogeny of 54 representative strains of species in the family Pasteurellaceae as determined by comparison of $16 \mathrm{~S}$ rRNA sequences. J. Bacteriol. 174:2002-2013.

4. Dorch, M., and E. Stackebrandt. 1992. Some modifications in the procedure of direct sequencing of PCR amplified 16S rDNA. J. Microbiol. Methods 16:271-279.

5. Eigelsbach, H. T., and C. M. Downs. 1961. Prophylactic effectiveness of live and killed tularemia vaccines. I. Production of vaccine and evaluation in the white mouse and guinea pig. $J$. Immunol. 87:415-425.

6. Eigelsbach, H. T., and V. G. McGann. 1984. Gram-negative aerobic cocci, p. 394-399. In N. R. Krieg and J. G. Holt (ed.), Bergey's manual of systemic bacteriology, vol. 1. Williams \& Wilkins Co., Baltimore.

7. Felsenstein, J. 1989. PHYLIP-phylogeny inference package (version 3.2). Cladistics 5:164-166.

8. Fitch, W. M., and E. Margoliash. 1967. Construction of phylogenetic trees: a method based on mutational distances as estimated from cytochrome c sequences is of general applicability. Science 155:279-284.

9. Forsman, M., G. Sandström, and B. Jaurin. 1990. Identification of Francisella species and discrimination of type A and type B strains of $F$. tularensis by $16 \mathrm{~S}$ rRNA analysis. Appl. Environ. Microbiol. 56:949-955.

10. Fox, G. E., J. D. Wisotzkey, and P. Jurtshuk, Jr. 1992. How 
close is close: $16 \mathrm{~S}$ rRNA sequence identity may not be sufficient to guarantee species identity. Int. J. Syst. Bacteriol. 42:166-170.

11. Fry, N. K., S. Warwick, N. A. Saunders, and T. M. Embley. 1991. The use of $16 \mathrm{~S}$ ribosomal RNA analyses to investigate the phylogeny of the family Legionellaceae. J. Gen. Microbiol. 137:1215-1222.

12. Hollis, D. G., R. E. Weaver, A. G. Steigerwalt, J. D. Wenger, C. W. Moss, and D. J. Brenner. 1989. Francisella philomiragia comb. nov. (formerly Yersinia philomiragia) and Francisella tularensis biogroup novicida (formerly Francisella novicida) associated with human disease. J. Clin. Microbiol. 27:16011608.

13. Hultman, T., S. Ståhl, E. Hornes, and M. Uhlén. 1989. Direct solid phase sequencing of genomic and plasmid DNA using magnetic beads as a support. Nucleic Acids Res. 17:4937-4946.

14. Kimura, M. 1980 . A simple model for estimating evolutionary rates of base substitutions through comparative studies of nucleotide sequences. J. Mol. Evol. 16:111-120.

15. Marchette, N. J., and P. S. Nicholes. 1961. Virulence and citrulline ureidase activity of Pasteurella tularensis. J. Bacteriol. 82:26-32.

16. Marconi, R. T., and C. F. Garon. 1992. Phylogenetic analysis of the genus Borrelia: a comparison of North American and European isolates of Borrelia burgdorferi. J. Bacteriol. 174:241244.

17. Martinez-Murcia, A. J., S. Benloch, and D. Collins. 1992. Phylogenetic interrelationships of members of the genera Aeromonas and Plesiomonas as determined by $16 \mathrm{~S}$ ribosomal DNA sequencing: lack of congruence with results of DNA-DNA hybridizations. Int. J. Syst. Bacteriol. 42:412-421.

18. Moody, M. D., and C. M. Downs. 1955. Studies on tularemia. I. The relation between certain pathogenic and immunogenic properties of variants of Pasteurella tularensis. J. Bacteriol. 70:297-304.

19. Murray, R. G. E., D. J. Brenner, R. R. Colwell, P. de Vos, M.
Goodfellow, P. A. D. Grimont, N. Pfennig, E. Stackebrandt, and G. A. Zavarzin. 1990. Report of the Ad Hoc Committee on Approaches to Taxonomy within the Proteobacteria. Int. J. Syst. Bacteriol. 40:213-215.

20. Olsufiev, N. G., O. S. Emelyanova, and T. N. Dunayeva. 1959. Comparative study of strains of Bacterium tularense. II. Evaluation of criteria of virulence of Bacterium tularense in the old and the new world and their taxonomy. J. Hyg. Epidemiol. Microbiol. Immunobiol. 3:138-149.

21. Sambrook, J., E. F. Fritsch, and T. Maniatis. 1989. Molecular cloning: a laboratory manual, 2nd ed. Cold Spring Harbor Laboratory Press, Cold Spring Harbor, N.Y.

22. Sandström, G., A. Sjöstedt, M. Forsman, N. V. Pavlovich, and B. N. Mishankin. 1992. Characterization and classification of strains of Francisella tularensis isolated in the Central Asian focus of the Soviet Union and in Japan. J. Clin. Microbiol. 30:172-175.

23. Sanger, F., F. Nicklen, and A. R. Coulsen. 1977. DNA sequencing with chain termination inhibitors. Proc. Natl. Acad. Sci. USA 74:5463-5469.

24. Stackebrandt, E., R. G. E. Murray, and H. G. Trüper. 1988. Proteobacteria classis nov., a name for the phylogenetic taxon that includes the "purple bacteria and their relatives." Int. J. Syst. Bacteriol. 38:321-325.

25. Tärnvik, A. 1989. Nature of protective immunity to Francisella tularensis. Rev. Infect. Dis. 11:440-451.

26. Weisburg, W. G., M. E. Dobson, J. E. Samuel, G. A. Dasch, L. P. Mallavia, O. Baca, L. Mandelco, J. E. Sechrest, E. Weiss, and C. R. Woese. 1989. Phylogenetic diversity of the rickettsiae. J. Bacteriol. 171:4202-4206.

27. Weiss, E., G. A. Dasch, and K.-P. Chang. 1984. Gram-negative aerobic cocci, p. 711-713. In N. R. Krieg and J. G. Holt (ed.), Bergey's manual of systemic bacteriology, vol. 1. Williams \& Wilkins Co., Baltimore. 